\title{
A solution to the flexible transmission benchmark by convex optimization
}

\author{
Gorka Galdos and Alireza Karimi *†‡
}

April 20, 2011

\begin{abstract}
The flexible transmission benchmark was proposed in the European Journal of Control to evaluate some robust digital control approaches in 1995. With progress in convex optimization algorithms new methods for robust controller design are developed. A recently proposed fixed-order robust controller design method is applied to this benchmark problem and the results are compared with the existing results. The proposed method is based on open-loop shaping with frequency-domain constraints on the closed-loop sensitivity functions in the Nyquist diagram. The method needs only the frequency response of the model and can consider the multimodel uncertainty. A controller is designed by a convex optimization algorithm that meets all the required performance specifications for the benchmark with the lowest controller complexity.
\end{abstract}

\section{Introduction}

There is an enormous number of methods for designing robust controllers in literature. These methods are based on different assumptions on the model uncertainty, use different control objectives and different mathematical tools for solving the robust control problems. Since a theoretical comparison of different methods is not possible, the benchmarking seems to be the best way to compare fairly different robust control approaches.

One of the well-known benchmarks for robust controller design was presented in the second European Control Conference in Rome (ECC 1995) and the results were published in a special issue of European Journal of Control (Vol. 1 No. $2,1995)$. The benchmark problem is to design a low-order robust controller for a flexible transmission system in three different loadings (Landau et al, 1995b). The model of system contains two low-damped resonance modes whose

\footnotetext{
*The authors are with the Laboratoire d'Automatique of Ecole Polytechnique Fédérale de Lausanne (EPFL), 1015 Lausanne, Switzerland.

$\dagger$ This research work is financially supported by the Swiss National Science Foundation under Grant No. 200020-107872.

$\ddagger$ Corresponding author: alireza.karimi@epfl.ch
} 
frequencies change drastically with load. The control specifications are given in terms of time-domain performance for tracking and disturbance rejection and frequency-domain performance in terms of the constraints on the magnitude of the sensitivity functions. The problem is challenging, because the large model uncertainty is located at low frequencies where the performance is required.

Many robust control design approaches have been already applied to the flexible transmission benchmark. Some of them are published in the special issue dedicated to the benchmark and the others ulteriorly with new progress in robust control approaches. At the time being the following solutions can be found in literature: four $H_{\infty}$ solutions (Jones and Limebeer, 1995; Walker, 1995; Kwakernaak, 1995; Ferreres and Fromion, 1999), two controllers based on Quantitative Feedback Theory (QFT) (Kidron and Yaniv, 1995; Nordin and Gutman, 1995), three controllers using pole placement with sensitivity shaping (Landau et al, 1995a; Landau and Karimi, 1998; Langer and Constantinescu, 1999), one Generalized Predictive Controller (GPC)(Decker et al, 1995), one fractional order controller by CRONE control (Oustaloup et al, 1995) and one model-free approach based on Iterative Feedback Tuning (IFT) (Hjalmarsson et al, 1995). Although all the conrollers stabilize the system and achieve good performance, only two controllers meet all required specifications for all loadings with relatively high-order controllers. The first controller that satisfied all specifications was a QFT controller with 20 parameters. Later on, a controller using convex optimization achieved the same performances with 16 parameters. Although the complexity of the controller was not among the benchmark constraints, it was added for evaluation of the solutions in a synthesis paper by the proposers of the benchmark problem (Landau et al, 1995b).

In the benchmark problem three discrete-time models of the flexible transmission in different loadings (no-load, half-load and full-load) are given. The main difficulty for most of the design methods is to deal with the multimodel uncertainty. Although this type of uncertainty is very common in industry, few methods dealing directly with multimodel uncertainty have been developed. Approximating the multimodel uncertainty by unstructured frequency domain uncertainty, leads usually to a conservative design and poor performance.

In this paper, the method presented in (Karimi and Galdos, 2010) is applied to the flexible transmission benchmark problem. Moreover, discrete-time controller design is explicitly presented and open-loop shaping is considered as main control criterion. This method can be used for robust fixed-order controller design using convex optimization for Linear Time Invariant (LTI) systems represented by nonparametric spectral models. In this approach the nonconvex set of fixed-order $H_{\infty}$ controllers is approximated by a set of convex constraints with respect to the parameters of a linearly parameterized controller. These convex constraints are obtained graphically from the Nyquist diagram of the open-loop system. The proposed method can be used for PID controllers as well as for higher order linearly parametrized controllers in discrete or continuous time. Additionally, this approach can treat directly the multimodel uncertainty which is not the case for the standard $H_{\infty}$ problem.

This paper is organized as follows: In Section 2 the flexible transmission 
benchmark problem is presented. Section 3 introduces the control design methodology based on the convex constraints in the Nyquist diagram. The solution to the benchmark problem is given in Section 4. Finally, some concluding remarks are given in Section 5 .

\section{Flexible Transmission Benchmark}

\section{$2.1 \quad$ System description}

The flexible transmission system is a laboratory setup designed and constructed in Laboratoire d'Automatique de Grenoble (INPG-CNRS), France. This system consists of three horizontal pulleys connected by two elastic belts. The input of the system is the reference position for the first pulley controlled by a DC motor in closed-loop. The output of the system is the position of the third pulley measured by a potentiometer. The schematic diagram of the system is given in Figure 1. The goal is to control the position of the third pulley which can be loaded with small disks. A PC is used to control the system with a sampling frequency of $f_{s}=20 \mathrm{~Hz}$ (sampling period of $50 \mathrm{~ms}$ ).

The system has two oscillatory modes with damping factors of less than 0.05 that vary significantly in different loadings. The discrete time models of the system for the no load, half load $(1.8 \mathrm{~kg})$ and full load $(3.6 \mathrm{~kg})$ configurations have been identified with a low magnitude Pseudo Random Binary Sequence (PRBS) input. The amplitude of the frequency characteristics is represented in Figure 2 where a normalized frequency for the discrete time system is used. In this diagram and all other frequency diagram of this paper the Nyquist frequency is divided by $f_{s}$, which leads to a discrete frequency range from 0 to $\pi$.

The discrete-time transfer functions of the system are given by:

$$
G_{i}\left(q^{-1}\right)=\frac{q^{-d} B_{i}\left(q^{-1}\right)}{A_{i}\left(q^{-1}\right)} \quad i=1,2,3
$$

where $q^{-1}$ is the backward shift operator and the pure time delay $d=2$ for all models. The corresponding identified and validated models are :

Unloaded model:

$$
\begin{aligned}
A_{1}\left(q^{-1}\right)= & 1-1.14833 q^{-1}+1.58939 q^{-2} \\
& -1.31608 q^{-3}+0.88642 q^{-4} \\
B_{1}\left(q^{-1}\right)= & 0.28261 q^{-1}+0.50666 q^{-2}
\end{aligned}
$$

Half loaded model:

$$
\begin{aligned}
A_{2}\left(q^{-1}\right)= & 1-1.99185 q^{-1}+2.20265 q^{-2} \\
& -1.84083 q^{-3}+0.89413 q^{-4} \\
B_{2}\left(q^{-1}\right)= & 0.1027 q^{-1}+0.18123 q^{-2}
\end{aligned}
$$

Fully loaded model:

$$
\begin{aligned}
A_{3}\left(q^{-1}\right)= & 1-2.09679 q^{-1}+2.31962 q^{-2} \\
& -1.93353 q^{-3}+0.87129 q^{-4} \\
B_{3}\left(q^{-1}\right)= & 0.06408 q^{-1}+0.10407 q^{-2}
\end{aligned}
$$




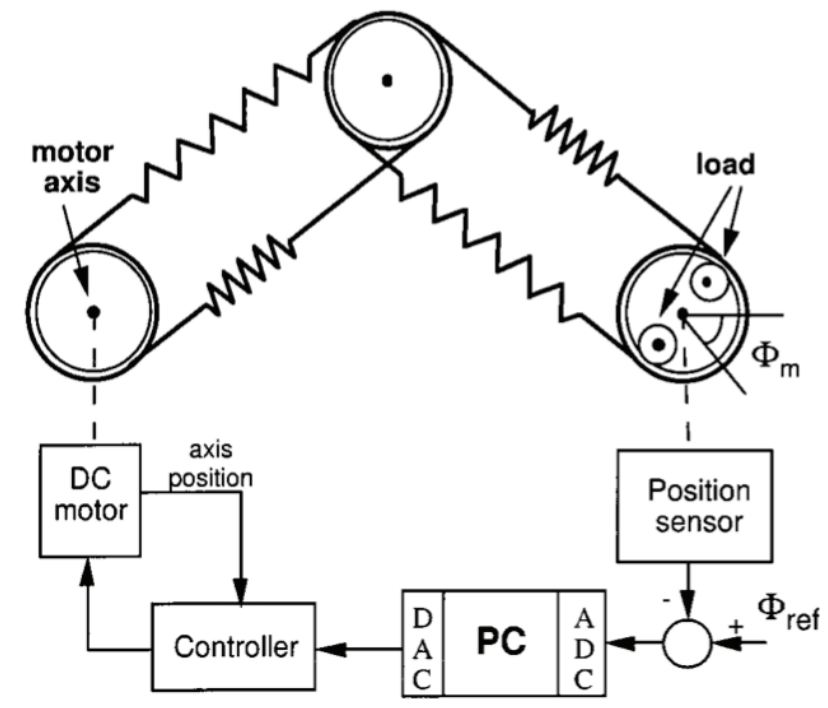

Figure 1: Schematic diagram of the flexible transmission.

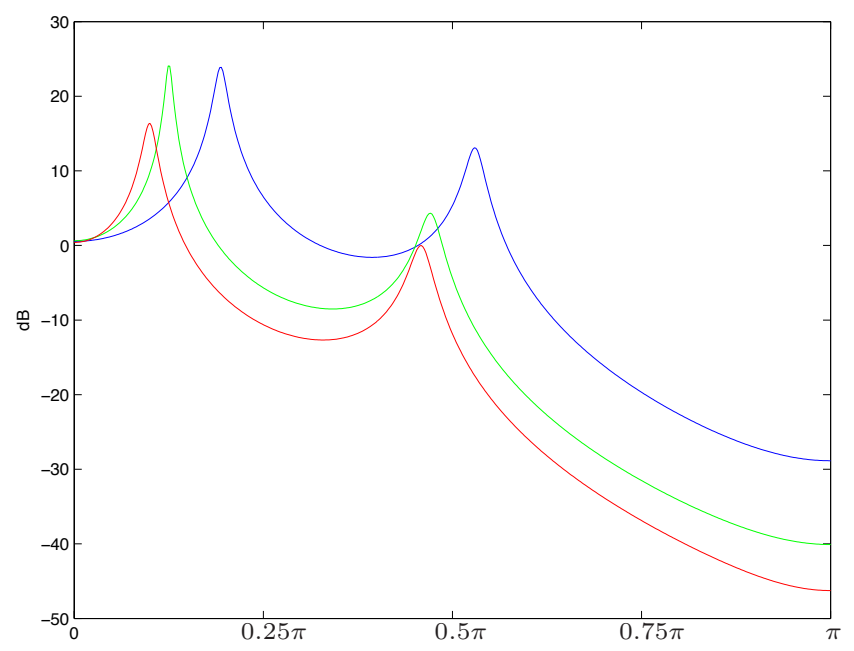

Figure 2: Frequency characteristics: Full loaded (red), Half loaded (green) and Unloaded (blue) 


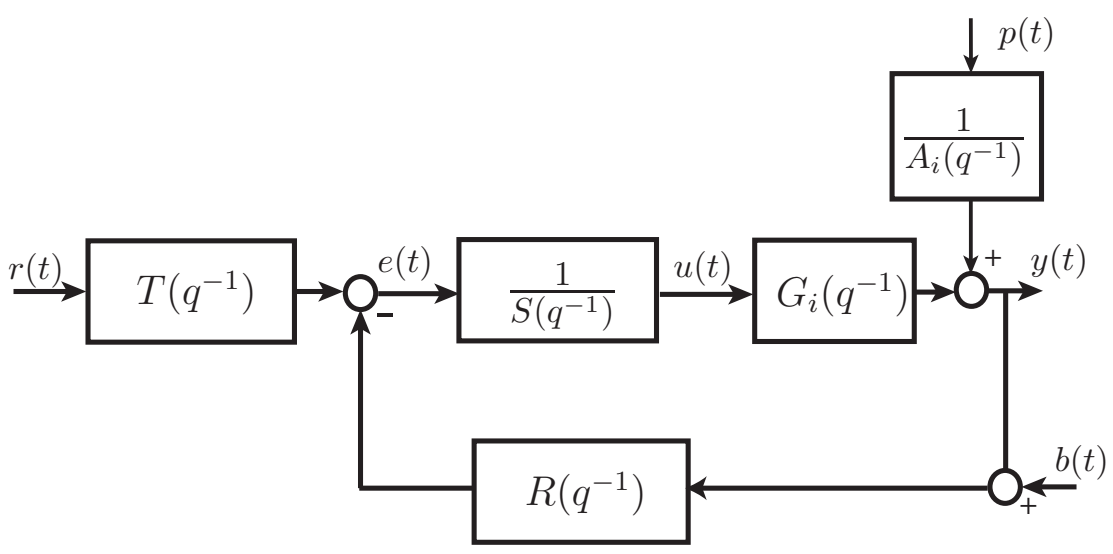

Figure 3: Structure of RST controller

\subsection{Benchmark specifications}

A discrete-time two-degree of freedom polynomial form RST controller (see Fig.3) has to be designed. The canonical form of the RST controller is given by:

$$
S\left(q^{-1}\right) u(t)=T\left(q^{-1}\right) r(t)-R\left(q^{-1}\right) y(t)
$$

where $u(t)$ is the plant input, $y(t)$ the plant output, $r(t)$ the desired reference and R,S,T are polynomials in $q^{-1}$.

The controller should be designed to satisfy the following specifications:

1. A rise time ( $90 \%$ of the final value) of less than $1 \mathrm{~s}$.

2. Overshoot of less than $10 \%$.

3. Rejection of $90 \%$ of the output disturbance $p(t)$ filtered by $1 / A_{i}$ in less than $1.2 \mathrm{~s}$.

4. Perfect rejection of a constant disturbance (using integral action).

5. Disturbance attenuation at low frequencies (less than $0.2 \mathrm{~Hz}$ ). It means that the gain of the output sensitivity function:

$$
\mathcal{S}_{i}\left(q^{-1}\right)=\frac{A_{i}\left(q^{-1}\right) S\left(q^{-1}\right)}{A_{i}\left(q^{-1}\right) S\left(q^{-1}\right)+q^{-d} B_{i}\left(q^{-1}\right) R\left(q^{-1}\right)}
$$

should be less than one, for frequencies less than $0.2 \mathrm{~Hz}$ (or $0.02 \pi$ of normalized frequency).

6. Maximum of the output sensitivity function less than $6 \mathrm{~dB}$ (modulus margin greater than 0.5).

7. A delay margin of at least $40 \mathrm{~ms}$ (0.8 of sampling periode). 
8. A maximum value of less than $10 \mathrm{~dB}$ for the input sensitivity function defined by:

$$
\mathcal{U}_{i}\left(q^{-1}\right)=\frac{A_{i}\left(q^{-1}\right) R\left(q^{-1}\right)}{A_{i}\left(q^{-1}\right) S\left(q^{-1}\right)+q^{-d} B_{i}\left(q^{-1}\right) R\left(q^{-1}\right)}
$$

at high frequencies (between 8 to $10 \mathrm{~Hz}$ ).

\section{Robust Controller Design in Nyquist Diagram}

\subsection{Class of models}

The class of causal stable discrete-time LTI-SISO systems with bounded infinity norm is considered. It is assumed that the plant model belongs to a set $\mathcal{G}$ containing $m$ spectral models :

$$
\mathcal{G}=\left\{G_{1}\left(e^{-j \omega}\right), G_{2}\left(e^{-j \omega}\right), \ldots, G_{m}\left(e^{-j \omega}\right) ; \quad \omega \in[0, \pi]\right\}
$$

This type of models can be obtained from a parametric model or by spectral analysis from a set of input/output data.

In the sequel, for the sake of simplicity, we develop the method for only one nominal discrete-time model $G \in \mathcal{G}$. However, it will be shown that the results are applicable to the multimodel case as well.

\section{$3.2 \quad$ Class of controllers}

Linearly parameterized controllers are given by :

$$
K\left(z^{-1}, \rho\right)=\rho^{T} \phi\left(z^{-1}\right)
$$

where

$$
\begin{gathered}
\rho^{T}=\left[\rho_{1}, \rho_{2}, \ldots, \rho_{n}\right], \\
\phi^{T}\left(z^{-1}\right)=\left[\phi_{1}\left(z^{-1}\right), \phi_{2}\left(z^{-1}\right), \ldots, \phi_{n}\left(z^{-1}\right)\right],
\end{gathered}
$$

$n$ is the number of controller parameters and $\phi_{i}\left(z^{-1}\right)$ are stable discrete-time transfer functions chosen from a set of orthogonal basis functions. Consider for example the Laguerre basis (Heuberger et al, 2005):

$$
\phi_{i}\left(z^{-1}\right)=\frac{\sqrt{1-\xi^{2}} z^{-1}}{1-a z^{-1}}\left[\frac{z^{-1}-\xi}{1-\xi z^{-1}}\right]^{i-1}
$$

with $|\xi|<1$ or a simpler FIR (Finite Impulse Response) basis:

$$
\phi_{i}\left(z^{-1}\right)=z^{-i+1}
$$

It is clear that for a low-order controller, the choice of basis functions becomes important. However, the influence of this choice, e.g. $\xi$ for Laguerre basis, is 
reduced when the controller order increases. An algorithm to choose the basis functions is proposed in (Karimi and Galdos, 2010).

The main property of this parameterization is that every point on the Nyquist diagram of the open-loop transfer function $L\left(e^{-j \omega}, \rho\right)$ can be written as a linear function of the controller parameters $\rho$ :

$$
L\left(e^{-j \omega}, \rho\right)=K\left(e^{-j \omega}, \rho\right) G\left(e^{-j \omega}\right)=\rho^{T} \phi\left(e^{-j \omega}\right) G\left(e^{-j \omega}\right)
$$

\subsection{Control criterion}

The control objective is open-loop shaping by minimizing the following criterion:

$$
J(\rho)=\left\|L(\rho)-L_{d}\right\|_{2}^{2}
$$

where $L_{d}\left(z^{-1}\right)$ is the desired open-loop transfer function. This criterion is a quadratic function with respect to the controller parameters and therefore can be used in a convex optimization problem. It should be noted that according to the function approximation theorem based on orthogonal basis functions, this norm can be made arbitrarily small by increasing the number of controller parameters (Heuberger et al, 2005).

The two-norm can be approximated when only a finite number $N$ of frequency points are available. In this case we have

$$
J(\rho)=\sum_{k=1}^{N}\left|L\left(e^{-j \omega_{k}}, \rho\right)-L_{d}\left(e^{-j \omega_{k}}\right)\right|^{2}
$$

Note that since the criterion is defined in the frequency domain, we need only the frequency response of $L_{d}$ which can be computed from a discrete-time or a continuous-time transfer function (i.e., $L_{d}\left(e^{-j \omega_{k}}\right)$ can be replaced by $\left.L_{d}(j \omega)\right)$.

The choice of $L_{d}$ is related to control specifications. In general, it should have high gain at low frequencies and low gain at high frequencies. A simple choice is the frequency response of an integrator : $L_{d}=\omega_{c} /(j \omega)$, where $\omega_{c}$ is the desired crossover frequency that determines the desired closed-loop bandwidth. An alternative is to choose $L_{d}$ based on a desired reference model $M$ for the closed-loop system. In this case we have $M=L_{d} /\left(1+L_{d}\right)$ which leads to $L_{d}=M /(1-M)$.

\subsection{Frequency-domain constraints}

It is clear that minimizing the criterion (10) does not lead necessarily to a stabilizing controller. The stability, robustness and some other performances can be represented by some infinity-norm constraints on the closed-loop transfer functions. The basic idea of a new approach to fixed-order $H_{\infty}$ controller design for systems represented by nonparametric frequency-domain models (Karimi and Galdos, 2010) is used to define these constraints. Although this method can be applied to continuous-time and unstable systems, here for simplicity of presentation only discrete time models and stable systems are considered. 
In the benchmark problem some constraints on the magnitude of the output sensitivity function

$$
\mathcal{S}\left(q^{-1}, \rho\right)=\left[1+L\left(q^{-1}, \rho\right)\right]^{-1}
$$

and input sensitivity function

$$
\mathcal{U}\left(q^{-1}, \rho\right)=K\left(q^{-1}, \rho\right)\left[1+L\left(q^{-1}, \rho\right)\right]^{-1}
$$

are imposed. These constraints can be represented in a general form as:

$$
\begin{aligned}
& \left|W_{1}\left(e^{-j \omega}\right) \mathcal{S}\left(e^{-j \omega}, \rho\right)\right|<1 \quad \forall \omega \\
& \left|W_{2}\left(e^{-j \omega}\right) \mathcal{U}\left(e^{-j \omega}, \rho\right)\right|<1 \quad \forall \omega
\end{aligned}
$$

where $W_{1}\left(e^{-j \omega}\right)$ and $W_{2}\left(e^{-j \omega}\right)$ are weighting functions. Multiplying these constraints by $\left|1+L\left(e^{-j \omega}, \rho\right)\right|$ gives:

$$
\begin{gathered}
\left|W_{1}\left(e^{-j \omega}\right)\right|<\left|1+L\left(e^{-j \omega}, \rho\right)\right| \quad \forall \omega \\
\left|W_{2}\left(e^{-j \omega}\right) K\left(e^{-j \omega}, \rho\right)\right|<\left|1+L\left(e^{-j \omega}, \rho\right)\right| \quad \forall \omega
\end{gathered}
$$

These constraints represent a nonconvex set. The following Theorem is a special case of Theorem 1 in (Karimi and Galdos, 2010) and represents a convex inner approximation of this nonconvex set.

Theorem 1 Consider a nonparametric model $G \in \mathcal{G}$ and a linearly parameterized controller $K\left(z^{-1}, \rho\right)$ defined in (3). This controller stabilizes $G$ and satisfies the nonconvex constraints in (13) and (14) if

$$
\begin{gathered}
\left|W_{1}\left(e^{-j \omega}\right)\left[1+L_{d}\left(e^{-j \omega}\right)\right]\right|-R_{e}\left\{\left[1+L_{d}^{*}\left(e^{-j \omega}\right)\right]\left[1+L\left(e^{-j \omega}, \rho\right)\right]\right\}<0 \quad \forall \omega \\
\left|W_{2}\left(e^{-j \omega}\right) K\left(e^{-j \omega}, \rho\right)\left[1+L_{d}\left(e^{-j \omega}\right)\right]\right|- \\
R_{e}\left\{\left[1+L_{d}^{*}\left(e^{-j \omega}\right)\right]\left[1+L\left(e^{-j \omega}, \rho\right)\right]\right\}<0 \quad \forall \omega
\end{gathered}
$$

where $L_{d}\left(e^{-j \omega}\right)$ should not encircle the critical point and $L_{d}^{*}\left(e^{-j \omega}\right)$ is its complex conjugate.

Proof: The proof is based on the fact that the real part of a vector is less than or equal to its magnitude, so we have:

$$
R_{e}\left\{\left[1+L_{d}^{*}\left(e^{-j \omega}\right)\right]\left[1+L\left(e^{-j \omega}, \rho\right)\right]\right\} \leq\left|\left[1+L_{d}^{*}\left(e^{-j \omega}\right)\right]\left[1+L\left(e^{-j \omega}, \rho\right)\right]\right|
$$

Replacing $R_{e}\left\{\left[1+L_{d}^{*}\left(e^{-j \omega}\right)\right]\left[1+L\left(e^{-j \omega}, \rho\right)\right]\right\}$ in (17) and (18) by the right hand side of the above inequality, the constraints in (15) and (16) are obtained respectively. On the other hand from (17), we have:

$$
R_{e}\left\{\left[1+L_{d}^{*}\left(e^{-j \omega}\right)\right]\left[1+L\left(e^{-j \omega}, \rho\right)\right]\right\}>0
$$

that means $\left[1+L_{d}^{*}\left(e^{-j \omega}\right)\right]\left[1+L\left(e^{-j \omega}, \rho\right)\right]$ will not encircle the origin. Since $L_{d}\left(e^{-j \omega}\right)$ and $L_{d}^{*}\left(e^{-j \omega}\right)$ do not turn around the critical point the winding number 
of $\left[1+L\left(e^{-j \omega}, \rho\right)\right]$ around the origin is also zero. This shows the stability of the closed-loop system with the controller $K\left(q^{-1}, \rho\right)$ and completes the proof.

The quality of this inner approximation depends on the desired open-loop frequency function $L_{d}\left(e^{-j \omega}\right)$. Assume that $\rho^{\circ}$ is a feasible point of the nonconvex set in (15) and (16). It means that there exists $0<\gamma \leq 1$ such that :

$$
\left|W_{1} \mathcal{S}\left(\rho^{\circ}\right)\right|<\gamma \quad \text { and } \quad\left|W_{2} \mathcal{U}\left(\rho^{\circ}\right)\right|<\gamma \quad \forall \omega
$$

On the other hand we have:

$$
R_{e}\left\{\left[1+L_{d}^{*}\right]\left[1+L\left(\rho^{\circ}\right)\right]\right\}=\left|1+L_{d}^{*} \| 1+L\left(\rho^{\circ}\right)\right| \cos \alpha
$$

where

$$
\alpha=\left|\angle\left[1+L\left(\rho^{\circ}\right)\right]-\angle\left[1+L_{d}\right]\right|
$$

is a function of $\omega$. Replacing the right hand side of (21) in (17) and (18), one obtains:

$$
\left|W_{1} \mathcal{S}\left(\rho^{\circ}\right)\right|<\cos \alpha \quad \text { and } \quad\left|W_{2} \mathcal{U}\left(\rho^{\circ}\right)\right|<\cos \alpha \quad \forall \omega
$$

It is clear that $\rho^{\circ}$ satisfy the above constraints if $\gamma<\cos \alpha$. In other words the phase difference between $L_{d}$ and $L\left(\rho^{\circ}\right)$ defines the level of conservatism of the convex approximation in Theorem 1. If $\alpha=0, \forall \omega$, there is no conservatism and smaller $\alpha$ leads to less conservatism.

\subsection{Optimization problem}

Consider the control criterion in (10) and convex constraints in (17) and (18) that represent a convex optimization problem with an infinite number of constraints (because the constraints are defined for all $\omega$ ). This problem can be converted to a Semi Definite Programming (SDP) problem if a frequency grid with $N$ samples between 0 and $\pi$ is used.

$$
\min _{\rho} \sum_{k=1}^{N}\left|L\left(e^{-j \omega_{k}}, \rho\right)-L_{d}\left(e^{-j \omega_{k}}\right)\right|^{2}
$$

Subject to:

$$
\begin{aligned}
&\left|W_{1}\left(e^{-j \omega_{k}}\right)\left[1+L_{d}\left(e^{-j \omega_{k}}\right)\right]\right|- R_{e}\left\{\left[1+L_{d}^{*}\left(e^{-j \omega_{k}}\right)\right]\left[1+L\left(e^{-j \omega_{k}}, \rho\right)\right]\right\}<0 \\
&\left|W_{2}\left(e^{-j \omega_{k}}\right) K\left(e^{-j \omega_{k}}, \rho\right)\left[1+L_{d}\left(e^{-j \omega_{k}}\right)\right]\right|- \\
& R_{e}\left\{\left[1+L_{d}^{*}\left(e^{-j \omega_{k}}\right)\right]\left[1+L\left(e^{-j \omega_{k}}, \rho\right)\right]\right\}<0
\end{aligned}
$$

for $k=1, \ldots, N$.

\section{Remarks:}


1. The solution of the above optimization problem will satisfy the constraints on the sensitivity functions and stabilizes the closed-loop system. However, it is not the optimal solution of the nonconvex problem because of the conservatism introduced in Theorem 1 for the convexification of the constraints.

2. Suppose that $\rho^{*}$ is the optimal solution of the nonconvex optimization problem, then $\rho^{*}$ will be a solution to the above convex optimization problem if the phase difference between $L\left(\rho^{*}\right)$ and $L_{d}$ is zero for all $\omega$.

3. Smaller phase difference between $L_{d}$ and $L\left(\rho^{*}\right)$ will reduce the conservatism of the convex approximation. This suggests an iterative approach in which $L_{d_{i}}=K\left(\rho_{i-1}\right) G$, where $L_{d_{i}}$ is the desired open-loop transfer function at $i$-th iteration and $\rho_{i-1}$ is the optimal solution from the previous iteration.

4. The number of frequency points should be sufficiently large such that $L\left(e^{j \omega}\right)$ between $\omega_{k}$ and $\omega_{k+1}$ is well approximated by linear interpolation of $L\left(e^{j \omega_{k}}\right)$ and $L\left(e^{j \omega_{k+1}}\right)$. The effects of this discretization on the stability and performance of this method are studied in (Galdos et al, 2010).

5. The above results can be extended to multimodel uncertainty by repeating the constraints for each model in the model set and minimizing the sum of two-norm errors for all models. This will be detailed in the next section.

\section{Solution to the Benchmark Problem}

The design procedure described in Section 3 is applied to the flexible transmission benchmark. We consider the following linearly parameterized two-degreeof-freedom RST controller :

$$
\begin{aligned}
R\left(q^{-1}\right) & =\rho_{1}+\rho_{2} q^{-1}+\ldots+\rho_{n} q^{-n+1} \\
S\left(q^{-1}\right) & =1-q^{-1} \\
T\left(q^{-1}\right) & =t_{0}
\end{aligned}
$$

For open-loop transfer function shaping this structure is equivalent to taking $K\left(z^{-1}\right)=R\left(z^{-1}\right) / S\left(z^{-1}\right)$ that leads to:

$$
K\left(z^{-1}\right)=\rho^{T} \phi\left(z^{-1}\right)
$$

with the following basis functions:

$$
\phi^{T}\left(z^{-1}\right)=\frac{1}{1-z^{-1}}\left[1, z^{-1}, z^{-2}, \ldots, z^{-n+1}\right]
$$

The fixed term $\left(1-q^{-1}\right)$ in $S\left(q^{-1}\right)$ is to assure the integral action of the controller (spec. 4). The time-domain performances (spec. 1,2 and 3) are tuned 
using the following reference model:

$$
M(s)=\frac{\omega_{n}^{2}}{s^{2}+2 \omega_{n} \xi s+\omega_{n}^{2}}
$$

This leads to the following desired open-loop transfer functions:

$$
L_{d_{i}}(s)=\frac{\omega_{n}^{2}}{s\left(s+2 \xi \omega_{n}\right)}
$$

for $\mathrm{i}=1,2,3$ (same desired open-loop transfer function for three models). Choosing $\omega_{n}=3.2 \mathrm{rad} / \mathrm{s}$ and $\xi=0.7$ gives a reference model with a rise time of $0.8 \mathrm{~s}$ and $5 \%$ overshoot which satisfies easily time-domain specifications (spec. 1 and 2).

It has been shown in (Landau and Karimi, 1998) that a delay margin of one sampling period is guaranteed if

$$
1-\left|1-e^{-j \omega}\right|^{-1}<\left|\mathcal{S}\left(e^{-j \omega}\right)\right|<1+\left|1-e^{-j \omega}\right|^{-1} \quad \forall \omega \in[0, \pi]
$$

The above constraint can be reformulated as :

$$
\left|W_{u}\left(e^{-j \omega}\right) \mathcal{S}\left(e^{-j \omega}\right)\right|<1 \quad \text { and } \quad\left|W_{l}\left(e^{-j \omega}\right) \mathcal{S}\left(e^{-j \omega}\right)\right|>1 \quad \forall \omega \in[0, \pi]
$$

where

$$
\left|W_{u}\left(e^{-j \omega}\right)\right|=\frac{\left|1-e^{-j \omega}\right|}{\left|1-e^{-j \omega}\right|+1} \quad \text { and } \quad\left|W_{u}\left(e^{-j \omega}\right)\right|=\frac{\left|1-e^{-j \omega}\right|}{\left|1-e^{-j \omega}\right|-1}
$$

The delay margin constraints in (27) can be converted to linear constraints using (17) in Theorem 1. This will guarantee a delay margin of $50 \mathrm{~ms}$ for the controlled system which is more than the desired delay margin of $40 \mathrm{~ms}$. However, we did not add this constraint because this specification (spec. 7) was met even without this constraint.

Because of two very oscillatory modes in the plant models, the output step disturbance filtered by $1 / A_{i}$ will be very oscillatory such that spec. 3 cannot be met. The disturbance rejection time can be reduced indirectly by adding a bound on the infinity-norm of the closed-loop transfer function between disturbance and output, $\mathcal{S}_{i} / A_{i}$. This constraint can be represented by :

$$
\left\|\frac{\mathcal{S}_{i}}{A_{i}}\right\|_{\infty}<\gamma_{i}
$$

and be considered in the proposed approach by taking a performance filter $W_{S_{i}}=1 /\left(\gamma_{i} A_{i}\right)$.

In addition, a performance filter

$$
W_{1}\left(e^{-j \omega}\right)= \begin{cases}1 & \text { for } 0<\omega \leq 0.02 \pi \\ 1 / 10^{6 / 20}=0.5 & \text { for } 0.02 \pi<\omega \leq \pi\end{cases}
$$


is chosen in order to assure a maximum of less than $6 \mathrm{~dB}$ (spec. 6) for the output sensitivity function (modulus margin of 0.5 ) and an attenuation band of $0.2 \mathrm{~Hz}$ (spec. 5). These performances can be given by one weighting function for each model defined as follows:

$$
W_{1 i}\left(e^{-j \omega}\right)=\max \left[\left|W_{1}\left(e^{-j \omega}\right)\right|,\left|W_{S_{i}}\left(e^{-j \omega}\right)\right|\right]
$$

The specification for the input sensitivity function $\left|\mathcal{U}_{i}\left(e^{-j \omega}\right)\right|<10 \mathrm{~dB}$ at high frequencies, between $8 \mathrm{~Hz}$ and $10 \mathrm{~Hz}$, is transformed to (spec. 8):

$$
\left\|W_{2} \mathcal{U}_{i}\right\|_{\infty}<1
$$

where

$$
W_{2}\left(e^{-j \omega}\right)= \begin{cases}0 & \text { for } 0<\omega \leq 0.8 \pi \\ 1 / 10^{10 / 20} & \text { for } 0.8 \pi<\omega \leq \pi\end{cases}
$$

The controller is tuned in two steps. In the first step the feedback controller is tuned based on the proposed method and by solving the following optimization problem:

$$
\min _{\rho} \sum_{i=1}^{3} \sum_{k=1}^{N}\left|\rho^{T} \phi\left(e^{-j \omega_{k}}\right) G_{i}\left(e^{-j \omega_{k}}\right)-L_{d_{i}}\left(e^{-j \omega_{k}}\right)\right|_{2}^{2}
$$

Subject to:

$$
\begin{aligned}
& \left|W_{1_{i}}\left(e^{-j \omega_{k}}\right)\left[1+L_{d_{i}}\left(e^{-j \omega_{k}}\right)\right]\right|- \\
& R_{e}\left\{\left[1+L_{d_{i}}^{*}\left(e^{-j \omega_{k}}\right)\right]\left[1+\rho^{T} \phi\left(e^{-j \omega_{k} h}\right) G_{i}\left(e^{-j \omega_{k}}\right)\right]\right\}<0 \\
& \left|W_{2_{i}}\left(e^{-j \omega_{k}}\right) \rho^{T} \phi\left(e^{-j \omega_{k}}\right)\left[1+L_{d_{i}}\left(e^{-j \omega_{k}}\right)\right]\right|- \\
& R_{e}\left\{\left[1+L_{d_{i}}^{*}\left(e^{-j \omega_{k}}\right)\right]\left[1+\rho^{T} \phi\left(e^{-j \omega_{k}}\right) G_{i}\left(e^{-j \omega_{k}}\right)\right]\right\}<0
\end{aligned}
$$

for $k=1, \ldots, N$ and $i=1,2,3$, where:

$$
\phi\left(e^{-j \omega_{k}}\right)=\frac{1}{1-e^{-j \omega_{k}}}\left[1, e^{-j \omega_{k}}, \ldots, e^{-(n-1) j \omega_{k}}\right]
$$

In the second step, the unique parameter in $T\left(q^{-1}\right)$ is taken equal to the sum of the parameters of $R\left(q^{-1}\right)$ giving a unit gain to the closed-loop system. The frequency response of the three models are computed at $\mathrm{N}=500$ equally spaced frequency points between 0 and $\pi$ (Nyquist frequency).

\subsection{Simulation Results}

For controller design, in the first step, $L_{d_{i}}$ in $(25)$ is used for all models and no constraint for disturbance rejection is considered $\left(\gamma_{i}\right.$ too large). Then the number of controller parameters $n$ is increased step by step to find a feasible solution for the optimization problem. For solving the convex optimization problem a 
Table 1: Performance of the controller

\begin{tabular}{|l|r|r|r|}
\hline Specification & No load & Half load & Full load \\
\hline Rise Time [s] & 0.80 & 0.75 & 0.70 \\
Overshoot[\%] & 3.87 & 3.92 & 6.56 \\
Dist. rejection [s] & 1.15 & 1.15 & 1.2 \\
Maximum $\mathcal{S}[\mathrm{dB}]$ & 5.93 & 4.41 & 5.12 \\
Delay Margin [ms] & 44 & 95 & 385 \\
Maximum $\mathcal{U}[\mathrm{dB}]$ & 9.20 & 9.86 & 9.99 \\
Attenuation band & 0.204 & 0.206 & 0.200 \\
{$[\mathrm{~Hz}]$} & & & \\
\hline
\end{tabular}

standar SDP solver, SeDuMi (Sturm, 1999), is used.The feasible solution satisfies almost all specifications except for disturbance rejection time. Then in the second step $\gamma_{i}$ is reduced gradually up to the point that all specifications are met. This procedure led to the first controller $K_{0}$ with $n=12$ (controller order equal to 11 ) and $\gamma_{i}=10^{28 / 20}$ (equal to $28 \mathrm{~dB}$ ) for $i=1,2,3$.

In order to reduce the complexity of the controller, a new iteration is carried out by changing the desired open-loop transfer function. The new desired open loop transfer function is defined as: $L_{d_{i}}\left(e^{-j \omega}\right)=K_{0}\left(e^{-j \omega}\right) G_{i}\left(e^{-j \omega}\right)$. It should be mentioned that the disturbance rejection time is the most critical specification to be met. So with this new $L_{d_{i}}$, a tighter bound for $\gamma_{i}$ is also considered. These bounds are chosen as follows: the infinity norm of $\mathcal{S}_{i} / A_{i}$ is measured for the initial controller that gives $21 \mathrm{~dB}, 23 \mathrm{~dB}$ and $25 \mathrm{~dB}$, respectively for $i=1,2,3$. Then in order to maintain the performance of the system for disturbance rejection, slightly higher bounds for the infinity norms of $\mathcal{S}_{i} / A_{i}$ are considered. This leads to $\gamma_{1}=22 d B, \gamma_{2}=24 d B$ and $\gamma_{3}=26 d B$. By running the convex optimization problem an 8-th order controller $K_{1}$ satisfying $100 \%$ of the specifications is obtained. The order is further reduced to $n=7$ by using $L_{d_{i}}\left(e^{-j \omega}\right)=K_{1}\left(e^{-j \omega}\right) G_{i}\left(e^{-j \omega}\right)$ that satisfies all specifications except the disturbance rejection time for the no-load model. This specification is also met by reducing $\gamma_{1}$ to $20 \mathrm{~dB}$. The final 6 th-order controller is given by:

$$
\begin{aligned}
R\left(q^{-1}\right)= & 0.632-1.781 q^{-1}+1.895 q^{-2}-1.062 q^{-3} \\
& +0.5247 q^{-4}-0.3399 q^{-5}+0.1887 q^{-6} \\
S\left(q^{-1}\right)= & \left(1-q^{-1}\right) \\
T\left(q^{-1}\right)= & 0.05733
\end{aligned}
$$

Figures 4 and 5 show that the specifications on the input sensitivity function $\mathcal{U}_{i}$ and output sensitivity function $\mathcal{S}_{i}$ are satisfied for the three models. Figure 6 shows the step and disturbance rejection responses. The details of achieved performance for the final controller are shown in Table 1.

Table 2 gives a joint evaluation of the performance and complexity of some controllers that have already been designed for the benchmark problem and compare them with the proposed controller. It can be observed that the proposed 


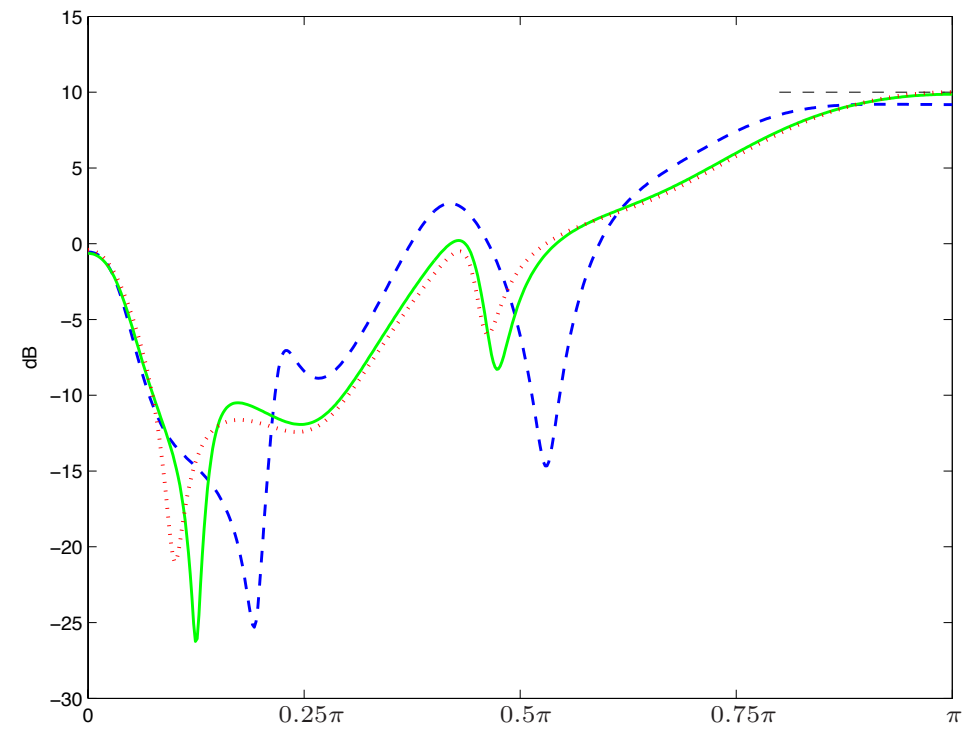

Figure 4: $\mathcal{U}$ of Unloaded (dashed, blue), Half loaded (solid, green) and Fully loaded (dashed-dotted, red) systems.

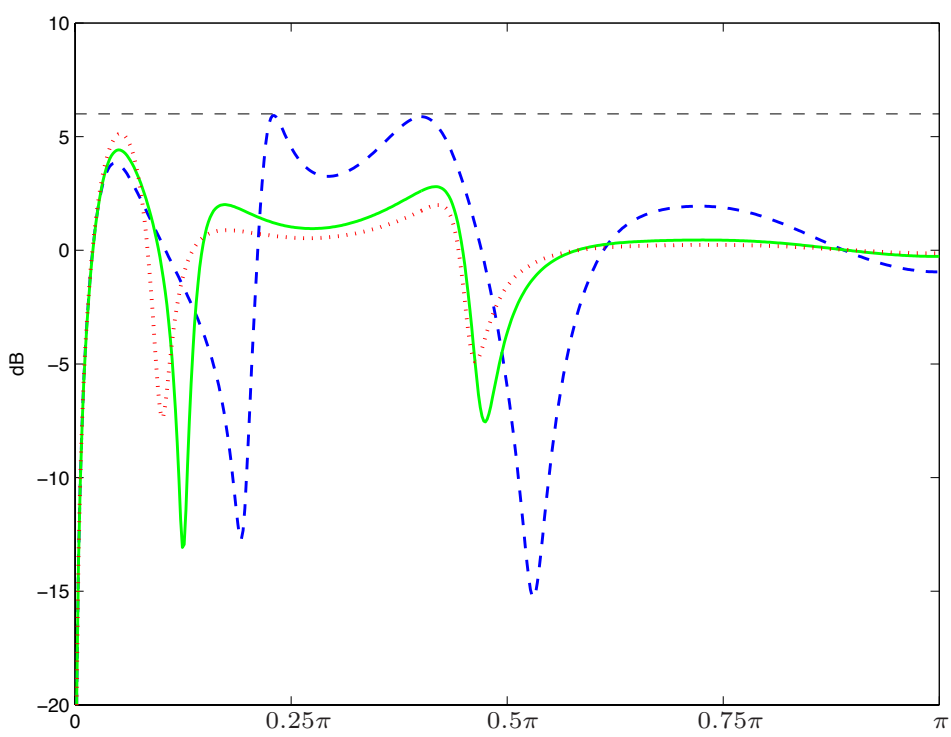

Figure 5: $\mathcal{S}$ of Unloaded (dashed, blue), Half loaded (solid, green) and Fully loaded (dashed-dotted, red) systems. 


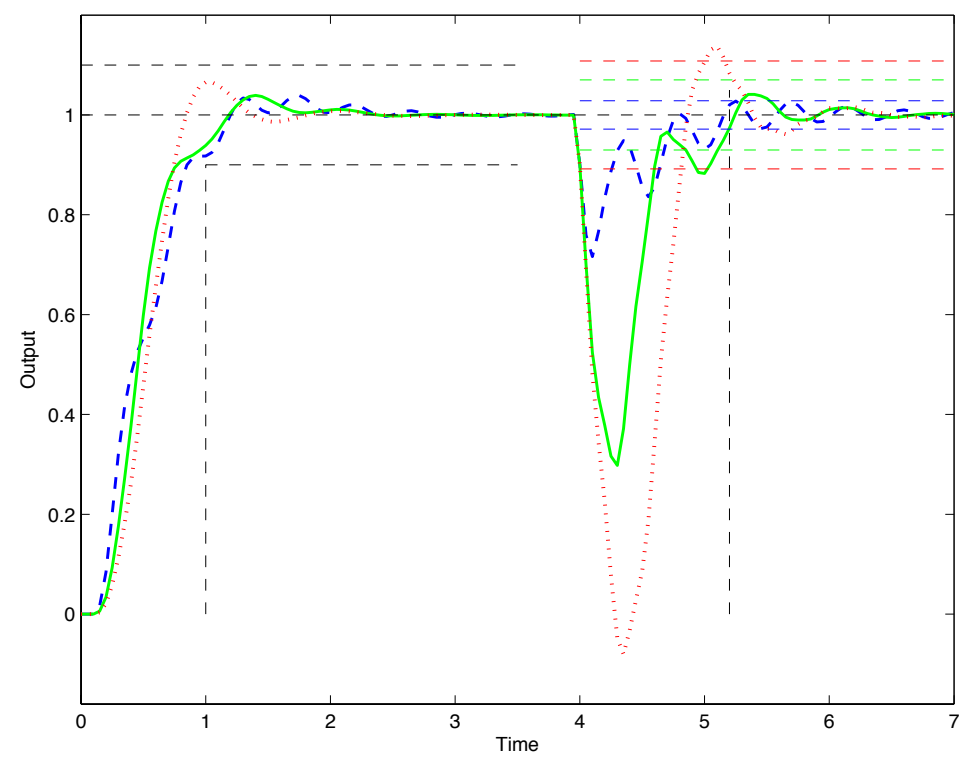

Figure 6: Step and disturbance rejection of Unloaded (dashed, blue), Half loaded (solid, green) and Fully loaded (dashed-dotted, red) systems. The straight dashed lines show the intervals in which the responses should be located.

controller meets all specifications with the lowest complexity.

\section{Conclusions}

A solution for the flexible transmission benchmark proposed in (Landau et al, 1995b) has been presented in this paper. To the best of knowledge of the authors, the proposed controller meets all the specifications with the lowest complexity amongst all controllers proposed for this system in literature. In this approach the multimodel uncertainty in the benchmark problem are directly considered in the design. Almost all specifications have been taken into account in the design procedure straightforwardly. Although the resulting controller has the smallest order among the benchmark solutions, it cannot be shown that there is no lower-order controller that can achieve the same performance.

\section{References}

Decker, C., Ehrlinger, A., Boucher, P. and Dumur, D., 1995, "Application of constrained receding horizon predictive control to a benchmark problem," European Journal of Control 1(2):157-165. 
Table 2: Comparison with other benchmark results

\begin{tabular}{ccc}
\hline & $\begin{array}{c}\text { Performance } \\
(\%)\end{array}$ & $\begin{array}{c}\text { Complexity } \\
\text { (order of R+S+T) }\end{array}$ \\
\hline Proposed approach & 100 & 7 \\
(Langer and Constantinescu, 1999) & 100 & 16 \\
(Nordin and Gutman, 1995) & 100 & 20 \\
(Ferreres and Fromion, 1999) & 98.80 & 11 \\
(Oustaloup et al, 1995) & 98.61 & 14 \\
(Kidron and Yaniv, 1995) & 97.71 & 9 \\
(Hjalmarsson et al, 1995) & 97.48 & 12 \\
(Landau et al, 1995a) & 97.12 & 35 \\
(Jones and Limebeer, 1995) & 94.38 & 16 \\
(Decker et al, 1995) & 91.82 & 15 \\
(Walker, 1995) & 72.35 &
\end{tabular}

Ferreres, G. and Fromion, V., 1999, " $H_{\infty}$ control for a flexible transmission system," European Journal of Control 5:185-192.

Galdos, G., Karimi, A. and Longchamp, R., 2010, "Robust controller design by convex optimization based on finite frequency samples of spectral models," In: 49th IEEE Conference on Decision and Control, Atlanta, USA, pp. 43174322 .

Heuberger, P., Van den Hof, P. and Wahlberg, B., 2005, Modelling and identification with rational basis functions, Springer-Verlag, London, UK.

Hjalmarsson, H., Gunnarsson, S. and Gevers, M., 1995, "Model free tuning of a robust regulator for a flexible transmission system," European Journal of Control 1(2):134-140.

Jones, N. W. and Limebeer, D. J. N., 1995, "A digital $H_{\infty}$ controller for a flexible transmission system," European Journal of Control 1(2):134-140.

Karimi, A and Galdos, G., 2010, "Fixed-order $H_{\infty}$ controller design for nonparametric models by convex optimization," Automatica 46(8):1388-1394.

Kidron, O. and Yaniv, O., 1995, "Robust control of uncertain resonant systems," European Journal of Control 1(2):104-112.

Kwakernaak, H., 1995, Symmetries in control system design, In: Isidori A (ed) Trends in Control, Springer Verlag, London.

Landau, I. D. and Karimi, A., 1998, "Robust digital control using pole placement with sensitivity function shaping method," International Journal of Robust and Nonlinear Control 8(2):191-210. 
Landau, I. D., Karimi, A., Voda, A. and Rey, D., 1995a, "Robust digital control of flexible transmission using the combined pole placement/sensitivity function shaping method," European Journal of Control 1(2):122-133.

Landau, I. D., Rey, D., Karimi, A., Voda, A. and Franco, A., 1995b, "A flexible transmission system as a benchmark for robust digital control," European Journal of Control 1(2):77-96.

Langer, J. and Constantinescu, A.,1999, "Pole placement design using convex optimisation criteria for the flexible transmission benchmark," European Journal of Control 5:193-207.

Nordin, M. and Gutman, P., 1995, "Digital QFT design for the benchmark problem," European Journal of Control 1(2):97-103.

Oustaloup, A., Mathieu, B. and Lanusse, P., 1995, "The CRONE control of resonant plants: Application to a flexible transmission," European Journal of Control 1(2):113-121.

Sturm, J. F., 1999, "Using SeDuMi 1.02, a Matlab toolbox for optimization over symmetric cones," Optimization Methods and Software 11:625-653.

Walker, D. J., 1995, "Control of a flexible transmission - a discrete time $H_{\infty}$ approach," European Journal of Control 1(2):141-147. 